organization. Our recent findings on site-specific strand-discontinuities in the genomic DNA of eukaryotes (2) also fit such a scenario.

This work has been supported by grants K128770 and GINOP-2.3.2-15-2016-00044 (Hungary).

(1) Imre at al., Sci Rep. 7(1):12734, 2017 (2) Hegedus et al., Nucleic Acids Res. 46(20):10649-10668, 2018

doi: http://dx.doi.org/10.7124/bc.0009A9

\section{The nucleosome: from structure to function through physics}

Andrew Fenley, Ramu Anandakrishnan, Yared Kidane, David Adamas, Alexey

V. Onufriev.

Virginia Tech, USA.

alexey@cs.vt.edu

Charge-altering PTMs in the globular histone core-including acetylation, phosphorylation, crotonylation, propionylation, butyrylation, formylation, and citrullination - can alter the strong electrostatic interactions between the oppositely charged nucleosomal DNA and the histone proteins and thus modulate accessibility of the nucleosomal DNA, affecting processes that depend on access to the genetic information, such as transcription. However, direct experimental investigation of the effects of these PTMs is very difficult. The goal is to make a step towards a unified theoretical model that connects post-translational modifications in the nucleosome with transcription in-vivo. Methods: Theory, Atomistic Simulations. Results: A physics-based framework is proposed that predicts the effect of charge-altering PTMs in the histone core, for most types of lysine charge-neutralizing PTMs including acetylation, and for phosphorylation. The predicted effect of chargealtering PTMs on DNA accessibility can vary dramatically, from virtually none to a strong, region-dependent increase in accessibility of the nucleosomal DNA; in some cases, e.g., $\mathrm{H} 4 \mathrm{~K} 44, \mathrm{H} 2 \mathrm{AK} 75$, and H2BK57, the effect is significantly stronger than that of the extensively studied acetylation sites such H3K56, H3K115 or H3K122. Proximity to the DNA is suggestive of the strength of the PTM effect, but there are many exceptions. For the vast majority of charge-altering PTMs, the predicted increase in the DNA accessibility should be large enough to result in a measurable modulation of transcription. However, a few possible PTMs, such as acetylation of H4K77, counterintuitively decrease the DNA accessibility, suggestive of the repressed chromatin. A structural explanation for the phenomenon is provided. Conclusions: Charge-altering post-translational modifications in the relatively unexplored globular histone core may provide a precision mechanism for controlling accessibility to the nucleosomal DNA. 